\title{
Strange quark content of the nucleon
}

\section{Ronald Babich, ${ }^{* a}$ Richard Brower, ${ }^{a b}$ Michael Clark, ${ }^{b}$ George Fleming ${ }^{c}$ James Osborn, ${ }^{d}$ and Claudio Rebbi ${ }^{a b}$}

${ }^{a}$ Department of Physics, Boston University, 590 Commonwealth Avenue, Boston, MA 02215, USA

${ }^{b}$ Center for Computational Science, Boston University,

3 Cummington Street, Boston, MA 02215, USA

${ }^{c}$ Department of Physics, Yale University,

New Haven, CT 06520, USA

${ }^{d}$ Argonne Leadership Computing Facility,

9700 S. Cass Avenue, Argonne, IL 60439, USA

E-mail: rbabich@bu.edu brower@bu.edu, mikec@bu.edu,

George.Flemingeyale.edu, osbornealcf.anl.gov, rebbi@bu.edu

We discuss the calculation of disconnected diagrams needed for determining the strange quark content of the nucleon on the lattice. We present results for the strange scalar form factor and the related parameter $f_{T s}$, which enters into the cross-section for the scattering of dark matter off nuclei in supersymmetric extensions of the standard model. In addition, we present results for the strange contribution to the nucleon's axial and electromagnetic form factors. The calculations were performed with two dynamical flavors of Wilson fermions on a $24^{3} \times 64$ anisotropic lattice with $a_{s} \approx 3 a_{t} \approx 0.11 \mathrm{fm}$ and $M_{\pi} \approx 400 \mathrm{MeV}$.

The XXVI International Symposium on Lattice Field Theory

July 14 - 19, 2008

Williamsburg, Virginia, USA

\footnotetext{
* Speaker.
} 


\section{Introduction}

As the lightest quark not participating in the valence sector, the strange quark plays a special role in our understanding of the structure of the nucleon. In recent years, there has been a vigorous experimental effort to determine the strange contribution to the nucleon's elastic electroweak form factors. In particular, a number of experiments have sought to measure the strange electric and magnetic form factors via parity-violating electron scattering [1], notably SAMPLE, A4, HAPPEX, and G0. Recent combined analyses [月, 看 find values for $G_{E}^{s}\left(Q^{2}\right)$ and $G_{M}^{s}\left(Q^{2}\right)$ that are small and consistent with zero in the range of momenta so far explored.

Also of interest is the strange axial form factor $G_{A}^{s}\left(Q^{2}\right)$, to which electron scattering experiments are relatively insensitive. At present, the best constraints come from the two-decades old neutrino scattering data of the E734 experiment at Brookhaven [ 4 ]. A recent analysis [5], combining these results with those of HAPPEX and G0, favors a negative value for $G_{A}^{s}\left(Q^{2}\right)$ in the range $0.45<Q^{2}<1.0 \mathrm{GeV}^{2}$. In time, a more precise determination should be possible with results from the new generation of neutrino scattering experiments (e.g., BooNE, MINER $v$ A). A special case is presented by the strange axial form factor at zero momentum transfer, $G_{A}^{s}(0)=\Delta s$, which may be identified with the strange quark contribution to the spin of the nucleon. This quantity is of particular importance, given the role sea quarks are thought to play in resolving the proton "spin crisis." In principle, it is accessible in deep inelastic scattering, where it is given by the first moment of the helicity-dependent structure function $\Delta s(x)$. In practice, however, determining the first moment requires an extrapolation of the experimental data to small values of $x$, where uncertainties are less under control. There is some tension between the two most recent analyses from HERMES [6, 7], which rely on different techniques; the former favors a negative value for $\Delta s$ while the latter finds a result consistent with zero, within somewhat larger uncertainties.

Unlike the strange electromagnetic and axial form factors, the strange scalar form factor $G_{S}^{s}\left(Q^{2}\right)$ is not directly accessible to experiment. At zero momentum transfer, this quantity corresponds to the strange scalar matrix element $\langle N|\bar{s} s| N\rangle$. Often considered in relation to the nucleonpion sigma term, it is an important parameter in models of the nucleon. It has also received attention, however, for the role it plays in the interpretation of dark matter experiments. Many models of TeV-scale physics, including most supersymmetric extensions of the standard model, yield a dark matter candidate (e.g., neutralino) that scatters from nuclei via Higgs exchange. The Higgs predominantly couples to strange quarks in the nucleon, and so $\langle N|\bar{s} s| N\rangle$ enters the cross-section through the quantity

$$
f_{T s}=\frac{m_{s}\langle N|\bar{s} s| N\rangle}{M_{N}}
$$

As emphasized recently in [8, 9], $f_{T s}$ is poorly known at present and represents the leading theoretical uncertainty in the interpretation of direct detection experiments.

Strange form factors have been explored on the lattice in a number of past investigations, mainly within the quenched approximation. For a partial list of references, we direct the reader to [10]. For some relevant interesting results presented at this conference, see [11, 12, 13].

\section{Method}

We begin by defining the basic correlation functions from which we will extract the strange 
form factors, starting with the usual two-point function for the proton. At momentum $\vec{q}$, this is given by

$$
G^{(2)}\left(t, t_{0} ; \vec{q}\right)=\left(1+\gamma_{4}\right)^{\alpha \beta} \sum_{\vec{x}} e^{i \vec{q} \cdot \vec{x}}\left\langle P^{\beta}(\vec{x}, t) \bar{P}^{\alpha}\left(\overrightarrow{0}, t_{0}\right)\right\rangle .
$$

Here $P^{\alpha}=\varepsilon_{a b c}\left(u_{a}^{T} C \gamma_{5} d_{b}\right) u_{c}^{\alpha}$ is the standard interpolating operator for the proton, with smeared quark fields, and $\left(1+\gamma_{4}\right)$ projects out the positive-parity state. Next, we define various three-point functions $G_{X}^{(3)}\left(t, t^{\prime}, t_{0} ; q^{2}\right)$, where $X=S, E, A$ correspond to the disconnected scalar, electric, and axial form factors, respectively. These are given by

$$
G_{S}^{(3)}\left(t, t^{\prime}, t_{0} ; \vec{q}\right)=\left(1+\gamma_{4}\right)^{\alpha \beta} \sum_{\vec{x}, \vec{x}^{\prime}} e^{i \vec{q} \cdot \vec{x}^{\prime}}\left\langle P^{\beta}(\vec{x}, t)\left[\bar{s} s\left(\vec{x}^{\prime}, t^{\prime}\right)-\left\langle\bar{s} s\left(\vec{x}^{\prime}, t^{\prime}\right)\right\rangle\right] \bar{P}^{\alpha}\left(\overrightarrow{0}, t_{0}\right)\right\rangle
$$

for the scalar,

$$
G_{E}^{(3)}\left(t, t^{\prime}, t_{0} ; \vec{q}\right)=\left(1+\gamma_{4}\right)^{\alpha \beta} \sum_{\vec{x}, \vec{x}^{\prime}} e^{i \vec{q} \cdot \vec{x}^{\prime}}\left\langle P^{\beta}(\vec{x}, t)\left[V_{4}\left(\vec{x}^{\prime}, t^{\prime}\right)-\left\langle V_{4}\left(\vec{x}^{\prime}, t^{\prime}\right)\right\rangle\right] \bar{P}^{\alpha}\left(\overrightarrow{0}, t_{0}\right)\right\rangle
$$

for the electric, and

$$
G_{A}^{(3)}\left(t, t^{\prime}, t_{0} ; \vec{q}\right)=\frac{1}{3} \sum_{i=1}^{3} \sum_{\vec{x}, \vec{x}^{\prime}} e^{i \vec{q} \cdot \vec{x}^{\prime}}\left[-i\left(1+\gamma_{4}\right) \gamma_{i} \gamma_{5}\right]^{\alpha \beta}\left\langle P^{\beta}(\vec{x}, t)\left[A_{i}\left(\vec{x}^{\prime}, t^{\prime}\right)-\left\langle A_{i}\left(\vec{x}^{\prime}, t^{\prime}\right)\right\rangle\right] \bar{P}^{\alpha}\left(\overrightarrow{0}, t_{0}\right)\right\rangle
$$

for the axial, where $V_{\mu}$ and $A_{\mu}$ are the point-split vector and axial currents. Note that we always employ the vacuum-subtracted value of the current, $[J(\vec{x}, t)-\langle J(\vec{x}, t)\rangle]$, even though this is only strictly necessary when $J$ is the scalar density, since the expectation value of the others vanish. Given finite statistics, however, and an inexact estimate of the trace, it is possible that using the vacuum-subtracted value gives reduced statistical errors. Empirically, we find that for the strange scalar and axial form factors at $q^{2}=0$, the two approaches give indistinguishable results. At larger momenta, however, uncertainties for the vacuum-subtracted quantities are noticeably smaller.

Inserting a complete set of states in Eq. (2.1) yields the spectral decomposition

$$
G^{(2)}\left(t, t_{0} ; \vec{q}\right)=\sum_{n} c_{n}(\vec{q}) e^{-E_{n}(\vec{q})\left(t-t_{0}\right)} .
$$

where the coefficients are given by

$$
c_{n}(\vec{q})=2\left(1+\frac{m_{n}}{E_{n}}\right) Z_{n}^{2}(\vec{q}) .
$$

Here $Z_{n}(\vec{q})$ is the amplitude for annihilating the $n$th state with our interpolating operator $(n=1$ corresponds to the nucleon itself). The momentum dependence in $Z_{n}$ arises because we utilize smeared quark sources for the nucleon. Performing a similar decomposition for a generic threepoint function, we find

$$
G_{X}^{(3)}\left(t, t^{\prime}, t_{0} ; \vec{q}\right)=\sum_{m, n} j_{n m}(\vec{q}) e^{-m_{n}\left(t-t^{\prime}\right)} e^{-E_{m}(\vec{q})\left(t^{\prime}-t_{0}\right)},
$$

where the coefficients $j_{n m}$ may in general be expressed in terms of some combination of form factors. In particular, the correlation functions given in Eqs. (2.2 2.4) have been defined such that a single form factor enters the coefficient $j_{11}$ for each case, according to

$$
j_{11}(\vec{q})=2\left(1+\frac{m_{1}}{E_{1}(\vec{q})}\right) Z_{1}(\overrightarrow{0}) Z_{1}(\vec{q}) G_{X}^{S}\left(q^{2}\right)
$$


for $X=S, E, A$, where $G_{X}^{s}\left(q^{2}\right)$ is the corresponding strange form factor of the nucleon. In terms of the coefficients $c_{n}$ extracted from the two-point function $G^{(2)}\left(t, t_{0} ; \vec{q}\right)$, this becomes

$$
j_{11}(\vec{q})=G_{X}^{S}\left(q^{2}\right) \sqrt{\frac{1}{2}\left(1+\frac{m_{1}}{E_{1}(\vec{q})}\right) c_{1}(\overrightarrow{0}) c_{1}(\vec{q})} .
$$

Our general strategy will be to fit the correlation functions $G_{X}^{(3)}\left(t, t^{\prime}, t_{0} ; \vec{q}\right)$ to Eq. (2.7), taking into account both the ground state nucleon and a single excited state. We may then extract the nucleon form factors from $j_{11}$ with input from the two-point function. In principle, one could also obtain form factors of the excited state from $j_{22}$, as well as transition form factors from $j_{12}$ and $j_{21}$. In practice, however, we expect these to absorb the contributions of still higher states and trust only the ground state form factors to be reliable.

The motivation for this approach will be discussed further as we present our results in the following section. Before turning there, however, we conclude this section with a few remarks on the calculation of the disconnected insertion. This represents a major challenge for the lattice since it requires the trace of the full Dirac propagator over color, spin, and spatial indices. Our approach for estimating this trace is described in [10]. Briefly, it involves inverting against a set of sources where each is nonzero on only a small number of sites, with these sites as widely separated as possible. In the parlance of the usual stochastic source method, this is equivalent to using a single noisy source with "extreme dilution." It provides a very accurate estimate of the trace, and since the small amount of contamination arising from off-diagonal terms is not gauge-invariant, the method is unbiased. On our $24^{3} \times 64$ lattice, we have chosen a dilution pattern where the smallest spatial separation between sites is $6 \sqrt{3} a_{s}$. With 864 (times 12 for color/spin) such sources per lattice, we obtain the trace over each of four time-slices.

In order to construct the nucleon correlators, we also calculate 64 propagators at the light quark mass per lattice, with one originating from each time-slice. We utilize gaussian smearing for the light quark propagators.

\section{Preliminary results}

The results presented here were calculated on a $24 \times 64$ anisotropic lattice, using an ensemble of 863 gauge configurations provided by the Hadron Spectrum Collaboration. These were generated with two dynamical flavors of unimproved Wilson fermions, with $M_{\pi} \approx 400 \mathrm{MeV}$ and $a_{s}=0.108(7) \mathrm{fm} \approx 3 a_{t}$. Given the anisotropy, this lattice has a relatively short extent in time, which has influenced our choice of method. Conventionally, one extracts the form factors by considering various ratios of the three- and two-point functions defined above. For example, at zero momentum transfer, one has

$$
R_{X}\left(t, t^{\prime}, t_{0} ; q^{2}=0\right)=\frac{G_{X}^{(3)}\left(t, t^{\prime}, t_{0} ; \overrightarrow{0}\right)}{G^{(2)}\left(t, t_{0} ; \overrightarrow{0}\right)} \rightarrow G_{X}^{s}\left(q^{2}\right),
$$

for large time separations. As discussed in the previous section, we have chosen instead to fit the three-point function that appears in the numerator of this ratio directly. The reasons are twofold. First, this allows us to avoid contamination from backward-propagating states, which are 


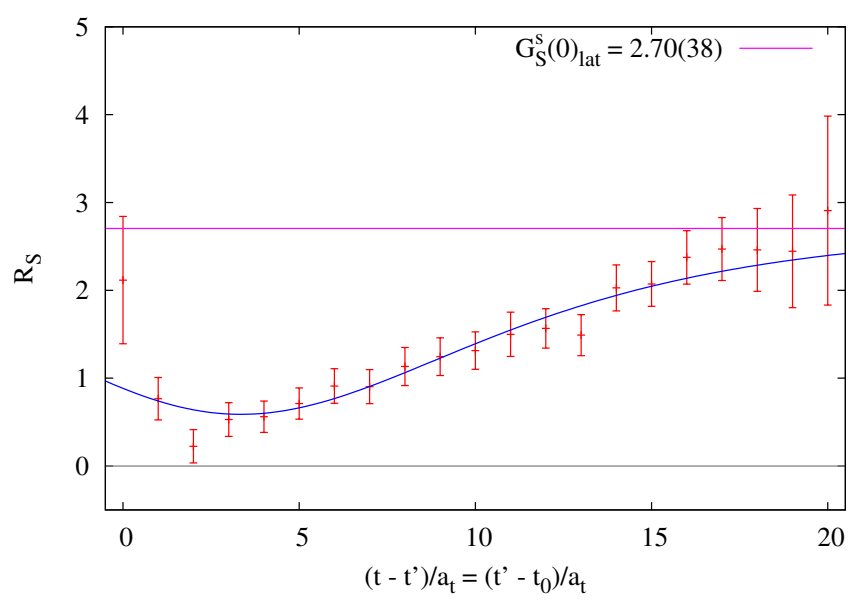

Figure 1: Results for the scalar form factor at $q^{2}=0$.

problematic due to the short temporal extent of our lattice. At the same time, it allows us to explicitly take into account the contribution of (forward-propagating) excited states.

Our fit of the scalar form factor at $q^{2}=0$ is shown in Figure 1. For the purpose of plotting, we have normalized our results by a fit to the two-point function. With this normalization, dominance of the ground state should manifest as a plateau at large times. The statistical error in the extracted form factor, $G_{S}^{S}(0)_{\text {lat }}=2.70(38)$ is determined by jackknife over the full fitting procedure. We note that in this preliminary analysis, we have treated the system symmetrically by requiring that the separation between the source and current insertion equal that between the insertion and the sink, i.e., $\left(t-t^{\prime}\right)=\left(t^{\prime}-t_{0}\right)$. It should be possible to reduce the statistical errors further by lifting this constraint. We expect that this will prove especially advantageous for the results at nonzero momentum transfer presented below, since these require a fit to a sum of four, rather than three, exponentials.

We have not yet attempted to quantify the systematic uncertainties. In addition to the usual systematics associated with the finite lattice spacing, finite volume, and the unphysically heavy mass of the light quarks, our approach is also sensitive to the choice of the fitting window in the fit of the two-point function. Recall that in order to extract the form factors from $G_{X}^{(3)}\left(t, t^{\prime}, t_{0} ; \vec{q}\right)$, we must first determine the coefficients $c_{n}(\vec{q})$ and masses/energies $E_{n}(\vec{q})$ from a fit to $G^{(2)}\left(t, t_{0} ; \vec{q}\right)$. Since we have access to a total of $863 \times 64=55,232$ nucleon correlators, these tend to be very welldetermined. The coefficients $c_{n}$ are somewhat sensitive to the choice of fitting window, however, and since they multiply the form factor in Eq. (2.9), this translates into a direct systematic error on the form factor, at perhaps the ten percent level.

Given our determination of the strange scalar matrix element, for the renormalization-invariant quantity $f_{T s}$, we estimate

$$
f_{T s}=\frac{m_{s}\langle N|\bar{s} s| N\rangle}{M_{N}}=0.51(8)(3),
$$

where we have inserted the physical nucleon mass for $M_{N}$. The second error is the uncertainty in relating this mass to the lattice scale, the first is statistical, and no other systematics have been 


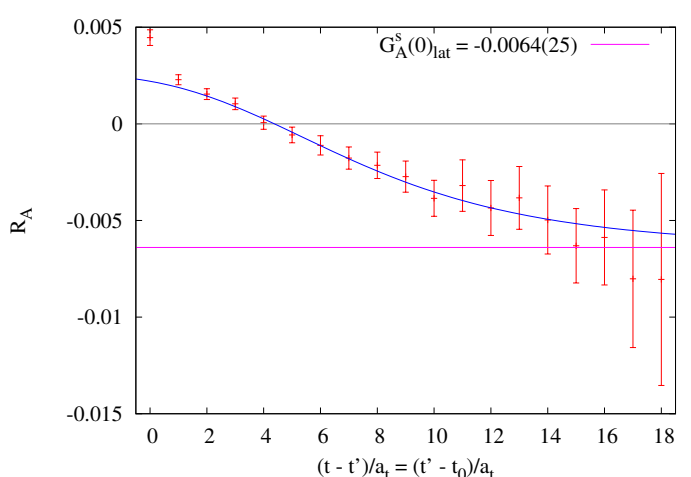

Figure 2: Results for the strange axial form factor at $q^{2}=0$.

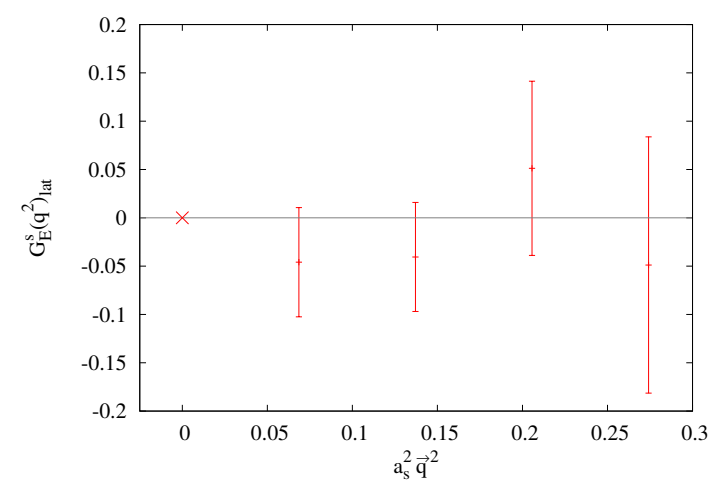

Figure 4: Strange electric form factor as function of momentum.

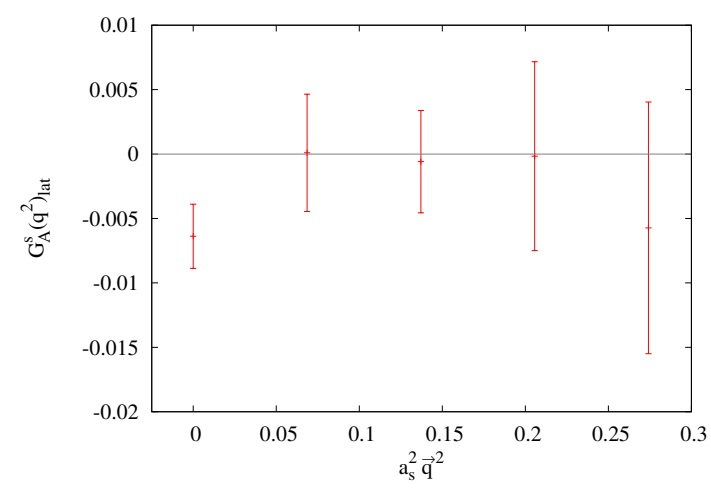

Figure 3: Strange axial form factor as function of momentum.

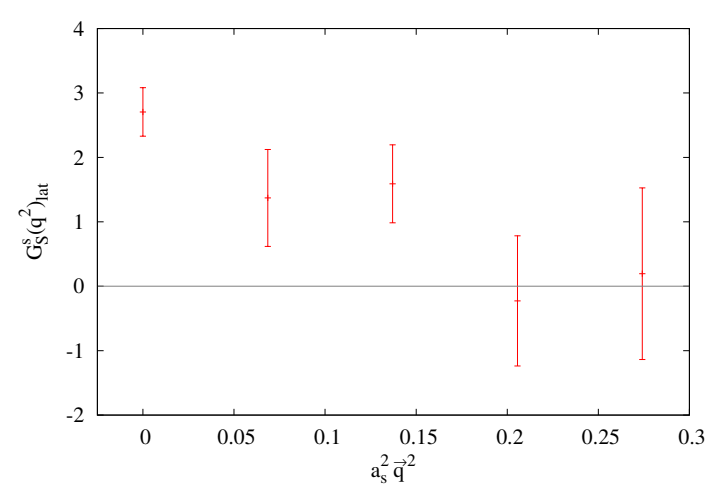

Figure 5: Strange scalar form factor as function of momentum.

taken into account. The bare quark mass $m_{s}$ is the naive mass appearing in the Wilson action, minus the critical mass, as determined from a (partially quenched) chiral extrapolation of the mass of the pseudoscalar. It is worth noting that the matrix element appearing in Eq. (3.2) was calculated for a world with a $400 \mathrm{MeV}$ pion. This is a consistent determination of the physical value only insofar as the matrix element $\langle N|\bar{s}| N\rangle$ is insensitive to the mass of the light quarks. As an alternative, we may work consistently in such a world by inserting our calculated value of the nucleon mass. In this case, the scale dependence drops out, and we find $f_{T S}=0.44(7)$.

Results for the strange axial form factor are shown in Figure2. As for $G_{S}^{s}(0)$, we note that our result $\Delta s=G_{A}^{s}(0)=-0.064(25)$ has not been renormalized and so may not be compared to the experimental results. It is noteworthy, however, that we find a value that is negative and distinct from zero at the level of 2.5 $\sigma$. In Figure 3, we show the momentum dependence of $G_{A}^{s}\left(q^{2}\right)$.

Finally, in Figure 4, we present our preliminary results for the strange electric form factor as a function of momentum. Note that since the strange quark does not contribute to electric charge of the nucleon, $G_{E}^{s}(0)$ must vanish. For completeness, the momentum dependence of the strange scalar form factor is shown in Figure 5 . 


\section{Acknowledgments}

This work was supported in part by US DOE grants DE-FG02-91ER40676 and DE-FC0206ER41440, NSF grants DGE-0221680 and PHY-0427646, and by the National Science Foundation through TeraGrid resources provided by the Texas Advanced Computing Center [14]. We also thank Boston University and Jefferson Lab for use of their scientific computing facilities.

\section{References}

[1] D. H. Beck and R. D. McKeown, Parity-violating electron scattering and nucleon structure, Ann. Rev. Nucl. Part. Sci. 51 (2001) 189-217, hep-ph/0102334].

[2] J. Liu, R. D. McKeown, and M. J. Ramsey-Musolf, Global Analysis of Nucleon Strange Form Factors at Low $Q^{2}$, Phys. Rev. C76 (2007) 025202, arXiv: 0706.0226 .

[3] R. D. Young, J. Roche, R. D. Carlini, and A. W. Thomas, Extracting nucleon strange and anapole form factors from world data, Phys. Rev. Lett. 97 (2006) 102002, [nucl-ex/0604010].

[4] L. A. Ahrens et al., Measurement of Neutrino - Proton and anti-neutrino - Proton Elastic Scattering, Phys. Rev. D35 (1987) 785.

[5] S. F. Pate, D. W. McKee, and V. Papavassiliou, Strange Quark Contribution to the Vector and Axial Form Factors of the Nucleon: Combined Analysis of GO, HAPPEx, and Brookhaven E734 Data, Phys. Rev. C78 (2008) 015207, arXiv:0805.2889.

[6] HERMES Collaboration, A. Airapetian et al., Precise determination of the spin structure function $g(1)$ of the proton, deuteron and neutron, Phys. Rev. D75 (2007) 012007, hep-ex/ 0609039 ].

[7] HERMES Collaboration, A. Airapetian et al., Measurement of Parton Distributions of Strange Quarks in the Nucleon from Charged-Kaon Production in Deep-Inelastic Scattering on the Deuteron, Phys. Lett. B666 (2008) 446-450, arXiv: 0803.2993 .

[8] A. Bottino, F. Donato, N. Fornengo, and S. Scopel, Size of the neutralino nucleon cross-section in the light of a new determination of the pion nucleon sigma term, Astropart. Phys. 18 (2002) 205-211, [hep-ph/0111229].

[9] J. R. Ellis, K. A. Olive, Y. Santoso, and V. C. Spanos, Update on the direct detection of supersymmetric dark matter, Phys. Rev. D71 (2005) 095007, [hep-ph/0502001].

[10] R. Babich et al., Strange quark contribution to nucleon form factors, PoS LAT2007 (2007) 139, arXiv:0710.5536.

[11] G. Bali, S. Collins, and A. Schafer, Hunting for the strangeness content of the nucleon, PoS LATTICE2008 (2008) 161, arXiv:0811.0807.

[12] T. Doi et al., Strangeness and glue in the nucleon from lattice QCD, PoS LATTICE2008 (2008) 163, [arXiv:0810.2482].

[13] H. Ohki et al., Calculation of the nucleon sigma term and strange quark content with two flavors of dynamical overlap fermions, PoS LATTICE2008 (2008) 126, arXiv: 0810.4223 .

[14] C. Catlett et al., TeraGrid: Analysis of Organization, System Architecture, and Middleware Enabling New Types of Applications, HPC and Grids in Action, Amsterdam (2007). 